\title{
El Área Metropolitana del Valle de Aburrá y las provincias. Retos de unión social y política
}

\section{The Área Metropolitana del Valle de Aburrá and the provinces. Challenges of political and social} union

\author{
Ángel Emilio Muñoz Cardona \\ Escuela Superior de Administración Pública-ESAP \\ angel@esap.gov.co
}

\begin{abstract}
Resumen
El fenómeno de conurbación generado por el crecimiento económico en el Valle de Aburrá obliga a los gobiernos a implementar politicas que contrarresten los efectos negativos de las economías de aglomeración; ¿qué está haciendo el gobierno para reducir los impactos de las inmigraciones en el territorio?, ¿por qué el Área Metropolitana posee reconocimientos internacionales de innovación en cultura? La investigación se basa en estadísticas sobre condiciones de vida de 10.725 familias, comunicaciones personales con funcionarios públicos de planeación municipal y en fuentes documentales de la administración pública. La investigación concluye que la región crece por la fuerza de la unión social, política y empresarial intermunicipal.
\end{abstract}

Palabras Clave: Área metropolitana, provincia, ciudades sustentables, ciudades innovadoras, ciudades de aglomeración y calidad de vida.

\begin{abstract}
The conurbation phenomenon generated by economic growth in the Aburra Valley forces governments to implement policies that counteract the negative effects of agglomeration economies, what is the government doing to reduce the impacts of immigration in the territory? why is the Metropolitan Area has international recognition of innovation in culture? The research is based on statistics on living conditions to 10,725 families, personal communications with public officials of municipal planning and in documentary sources of the public administration. The research concludes that the region grows by the force of social, political and business union of its municipalities.
\end{abstract}

Key Words: Metropolitan area, province, sustainable cities, innovative cities, agglomeration cities and quality of life.

Articulo: Recibido el 30 de julio de 2018 y aprobado el 15 de octubre de 2018.

\section{Cómo citar este articulo}

Muñoz Cardona, Á. E. (2019). El Área Metropolitana del Valle de Aburrá y las provincias. Retos de unión social y politica. Reflexión Política 21(41), pp. 175-189. DOI: https://doi.org/10.29375/01240781.3319 
"Las calles y sus aceras, los principales lugares públicos de una ciudad, son sus órganos más vitales. ¿Qué es lo primero que nos viene a la mente al pensar en una ciudad? Sus calles. Cuando las calles de una ciudad ofrecen interés, la ciudad entera ofrece interés; cuando presentan un aspecto triste, toda la ciudad parece triste"

Jane Jacobs

\section{Introducción}

Antioquia es una región montañosa, atravesada por dos ramales de la cordillera de los Andes que hicieron de ella, durante la época de la colonia, un lugar de difícil acceso y de imposible florecimiento económico por su inclinada topografia; sin embargo, desde mediados del siglo XIX al XXI se ha consolidado como uno de los departamentos mejor administrados, organizado y de mayor prosperidad en Colombia para sus 6.300.000 habitantes, ¿qué hipótesis explican tales logros? La investigación tiene dos objetivos fundamentales: 1) explicar las razones de crecimiento económico y de calidad de vida en el Departamento; 2) exponer la importancia de las provincias como reto para el control de los fenómenos de conurbación. En otras palabras, responder la pregunta: ¿cuáles son los retos que debe asumir la región para reducir los impactos negativos de las economías de aglomeración en el Área Metropolitana con más de 3.800.000 habitantes?

Para responder las preguntas de investigación, el estudio se dividió en tres partes; en la primera, se abordan 4 de las hipótesis más relevantes que explican el desarrollo de Antioquia; en la segunda, se aborda el desarrollo de Antioquia a partir de la conformación del Área Metropolitana y de las provincias; y en la tercera parte, se abordan los retos del Área Metropolitana a partir de su visión asociativa y de las metas estadísticas de condiciones de vida. Pero, antes de comenzar con la primera parte es necesario aclarar que no todo el que nace en Antioquia posee cultura paisa, ya que, por división política territorial en 1905, a raíz de la crisis política generada por los resultados de la guerra de los mil días que terminó con la pérdida de Panamá, se disolvieron los ocho Estados Unidos de Colombia y se conformaron los Departamentos. Al nuevo Departamento de Antioquia, le fueron asignados territorios de otras culturas y le fueron retirados otros de su misma idiosincrasia, proceso de separación político-administrativa que terminó en 1966.

Entre los asentamientos de otras civilizaciones, las más notables son las del departamento de Córdoba y del Chocó, -que dieron origen al Chilapo, unión del negro con el indígena- asentamientos que se han ido reforzando con inversiones para el crecimiento económico y de bienestar. Bajo el Plan Estratégico Antioquia Visión 2030 en el Golfo de Urabá se desarrollan tres puertos desde el 2017 en asociación público-privada con el sector bananero, Ocean Alliance, la Gobernación de Antioquia, la Universidad de Antioquia y la Escuela Superior de Administración Pública. Los puertos son: Puerto Antioquia, Pisisí en Turbo y Darien international Port en Necoclí.

Desde principios del siglo XVIII, los antioqueños de cultura paisa se asentaron principalmente en tierras aptas para el cultivo del café y del ganado (Restrepo, 2011). Dicha cultura se distingue por sus apellidos, gastronomía, acento lingüístico, religiosidad, capacidad emprendedora, organización territorial, habitacional y de unión político-cultural. Son parte de la cultura paisa más del $80 \%$ de los habitantes que conforman los Departamentos de Caldas, Risaralda, Quindío y Cartago en el Valle del Cauca.

Varias son las hipótesis que explican las diferencias de desarrollo y crecimiento económico de Antioquia; algunas evocan el carácter regionalista de su gente y su inclinación natural a ser empresarios y emprendedores; otras hipótesis fundamentan su explicación en el buen aprovechamiento de sus propias riquezas naturales. Sin embargo, la investigación concluye que la hipótesis más acertada para explicar el auge del desarrollo en Antioquia es la capacidad que tienen sus gentes, empresarios e instituciones políticas y sociales para trabajar unidas, en pos de un mismo ideal. Incluso desde antes de los inicios de la elección popular de alcaldes y gobernadores a finales de la década de los años 80s, en el Departamento se han venido trazando los Planes Estratégicos para el Desarrollo a 10 y 12 años (CEPAL y GTZ, 2000), tales como: Antioquia Visión Siglo XXI, Antioquia la Mejor Esquina de América, Antioquia Visión 2020, Antioquia Visión 2030, entre otros.

La investigación siguió los siguientes procesos metodológicos: primero, método histórico, con amplia consulta bibliográfica y de hemeroteca. Segundo, entrevistas a expertos de la planeación municipal del Área Metropolitana y del Departamento, bajo la metodología del "Focus Group" -economistas, ingenieros de 
ciudad y arquitectos-. Tercero, análisis de encuestas sobre Calidad de Vida bajo el método estadístico multivariado a 10.725 hogares del sector urbano en asocio con el Área Metropolitana del Valle de Aburrá con una confiabilidad del $98,5 \%$ y un margen de error del 1,5\%. Cuarto, grupos de discusión y análisis con el apoyo de las 11 alcaldías de la provincia de Cartama, el King's College London, la Universidad de Padova en Italia, el Departamento de Planeación de Antioquia, la Organización Internacional para la Migración (OIM), la Agencia de los Estados Unidos para el Desarrollo Internacional (USAID) y estudios doctorales realizados al Área Metropolitana dentro de la Escuela de Altos Estudios en Ciencias Sociales de París (EHESS).

Finalmente, el grupo de investigación consolidado en COLCIENCIAS agradecen a la ESAP por el apoyo financiero y a la OIM y la USAID por los reconocimientos dados a la investigación.

\section{Cuatro hipótesis sobre el desarrollo de Antioquia hasta 1986}

La gran ciudad del Área Metropolitana del Valle de Aburrá está integrada por 10 municipios que concentran 3.800.000 habitantes, la segunda ciudad más poblada de Colombia. Por ser una ciudad de aglomeración, en ella se asientan 134.775 microempresas, 13.048 pequeñas empresas, 3.469 medianas empresas y 1.109 grandes empresas, como lo enseñan los informes (Cámara de Comercio de Medellín para Antioquia, 2017; Cámara de Comercio Aburrá Sur, 2017).

La primera hipótesis que busca explicar la expansión del desarrollo industrial en Antioquia durante los siglos XIX y XX que marca el auge económico y social del Departamento, la expresa el inglés Roger Brew (2000); para el historiador la unión de capital de las familias antioqueñas fue el impulso del desarrollo industrial. En el siglo XIX dicha sociedad familiar fue la que facilitó el nacimiento de las sociedades anónimas y las tres primeras bolsas de valores del país -la Bolsa Popular de Medellín en 1901, la Bolsa Comercial de Medellín en 1903 y la Bolsa de Comercio de Medellín en 1929- Las familias se unían para aportar capital en inversiones de alto riesgo como fueron la creación de bancos y la explotación minera, por lo que Brew (2000) afirma:
En un medio inseguro y sin instituciones legales adecuadas, la unión familiar fue esencial, lo que facilitó la confianza de inversión en empresas demasiado grandes para los recursos de un solo individuo. Por otra parte, la familia podía recurrir a las reservas de capital y de conocimientos técnicos de todos sus miembros, quienes consideraban que la confianza mutua y la multiplicidad de actividades significaban la seguridad a largo plazo para todos. Así la familia, además de funcionar como un banco en beneficio de todos los parientes, cumplía las funciones de una institución de seguridad mutua y movilizaba el capital en los diferentes sectores económicos (pp. 81-82).

De igual manera, lo expresa la investigadora del Centro de Investigaciones Económicas (CIE) María Mercedes Botero Restrepo (1985), al afirmar que el surgimiento de los primeros bancos en Colombia se dio en Antioquia gracias a la unión de miembros de las familias antioqueñas que ponían sus capitales ganados en el comercio y en la caficultura, incluso sus tierras y el buen nombre que tenían servían de respaldo en la constitución de nuevos bancos y nuevas monedas. Afirma la economista que durante las épocas de crisis de la economía antioqueña de 1876 los tenedores de billetes acudían a los bancos a cambiarlos, la conversión y los pagos de intereses se cumplieron de manera tan estricta, que aumentó la confianza del público en la banca antioqueña (Botero, 1985, p. 101). Asimismo, ocurrió, a principios del siglo XX, con la quiebra temporal del Banco de Medellín, la cual fue rápidamente cubierta por el resto de los bancos de la ciudad con el fin de mantener la credibilidad de la moneda en todo el territorio y en todo el país para bien de la banca y de la economía antioqueña (Botero, 1985, p. 112).

Este respaldo de confianza y apoyo se refuerza con la creación del Grupo Empresarial Antioqueño GEA en 1978, con el fin de conservar la propiedad empresarial ante las amenazas de pérdida por inversión extranjera -no antioqueñosa través del mercado de acciones en los mercados bursátiles. Estrategia que fortaleció la inversión social de la empresa en la región (Londoño, 2005), y dinamizó el mercado bursátil nacional. En la actualidad en el índice de COLCAP, que mide las 20 empresas de mayor capitalización en Colombia, al menos 12 son del GEA.

La segunda hipótesis, formulada por el ingeniero industrial Nicanor Restrepo Santamaría, 
afirma que una de las características principales del desarrollo industrial en Antioquia se basó en la diversificación productiva y de inversión que hacían las familias en actividades complementarias. De igual manera, jugó un papel muy importante la administración profesional de las empresas, las cuales, por lo general, fueron dirigidas por gerentes ajenos a las familias y seleccionados por sus capacidades (Restrepo, 2011, p. 56). Hipótesis que se refuerza con la cultura del empresario antioqueño de aprender de quienes saben y tienen más experiencia; de allí que las asociaciones de empresarios antioqueños con industriales extranjeros funcionaba sin entregarse el manejo total de la empresa, pues el fin de dichas asociaciones era el de aprender nuevas formas productivas y de comercialización. Una vez se dominaba el proceso, los empresarios antioqueños buscaban comprar la participación del extranjero dentro de la empresa (Restrepo, 2011, pp. 59-60).

En Antioquia se observa que la propiedad de las empresas donde se han dado alianzas con extranjeros, con excepción de Almacenes Éxito, sigue estando controlada por los accionistas locales, y la administración continúa siendo ejercida por antioqueños que mantienen un papel activo en las diferentes actividades económicas, tanto nacionales como regionales, y que forman parte de las élites patronales de la región. (Restrepo, 2011, p. 60)

Fue la cultura de conservar la propiedad de las empresas y la disposición de aprender para mejorar lo que facilitó la capacidad competitiva y la permanencia de la gran empresa antioqueña en el medio. Principios administrativos que motivaron la unión empresarial del GEA, organización que hace parte de los concejos directivos en universidades que sirven de apoyo al crecimiento de sus 125 empresas $\mathrm{y}$ forman parte de su staff administrativo; se pueden nombrar dentro de estas: la Universidad EAFIT, el Instituto de Ciencias de la Salud CES y la Escuela de Ingeniería de Antioquia (Restrepo, 2011, p. 131). Del mismo modo, el GEA en asociación público-privado es un activo participante con ayudas de capital de riesgo y acompañamiento a jóvenes empresarios y emprendedores sin experiencia comercial en el ex- terior, a través del programa de acompañamiento empresarial Plan Padrino, Ruta N, Parque Explora, Innova y con prácticas empresariales y laborales a estudiantes próximos a graduarse de la Universidad de Antioquia, Universidad de Medellín y Universidad Pontificia Bolivariana, entre otros centros académicos de reconocida trayectoria en la región.

La tercera hipótesis, expuesta por los historiadores de la economía colombiana (Bejarano, 1984; Melo, 1991), se fundamenta en la riqueza minera y recursos naturales que ha poseído el departamento de Antioquia. La agreste topografía antioqueña dificultó el ingreso de los españoles durante la colonia, lo que facilitó a la región la acumulación de numerario en oro, la conservación de grandes minas de oro, carbón y hierro que facilitaron la inversión en vías para los ferrocarriles. De igual manera, el clima y la riqueza natural de sus suelos favorecieron la producción cafetera y la producción de energía eléctrica por las grandes caídas de agua. Pero, la cualidad más importante del empresariado antioqueño "es la fuerza del regionalismo que sumada al amor por el territorio han hecho del departamento uno de los más prósperos" (Melo, 1991, pp. 147-148).

Los dirigentes del departamento se sintieron muchas veces discriminados por los políticos y gobiernos nacionales, y con base en ello iniciaron movimientos multipartidistas orientados a extraer concesiones del gobierno nacional. De igual manera, la dirigencia antioqueña buscaba abrir el camino a la descentralización. (Melo, 1991, p. 147)

Sentir que, por partir de la misma dirigencia patronal, encontró eco en la dirigencia política y en el pueblo raso. Sentimientos que durante toda la historia han alimentado el llamado orgullo paisa de amor por su región, de mantener la propiedad y superioridad en el desarrollo de la industria nacional, y el de ofrecer a sus habitantes la mejor cobertura en servicios de infraestructura social. ${ }^{1}$ La fuerza del regionalismo alimentó y alimenta los sentimientos de unidad territorial más que los de exclusión o los del resentimiento; en otras palabras, los sentimientos de resiliencia o de amor propio para la prosperidad territorial. Pero, una de las razones que más alimentó el regionalismo fue la tenencia

$1 \quad$ El regionalismo en Antioquia lejos de ser un problema de racismo o de xenofobia es fundamentalmente una estrategia sociocultural de unión y de protección económica de sus empresas y de las propiedades de sus territorios. Es el medio por el cual se logra la solidaridad para el progreso social, político y económico, como lo afirma el ingeniero industrial Nicanor Restrepo Santamaría (2011) y Coenen-Huther (2004). 
de la tierra: cada campesino era dueño de su tajo de tierra y de lo producido por ella. Método de explotación económica basado en el minifundio con mano de obra libre, que para el siglo XIX y principios del siglo XX no existía en el resto del país; lo que ayudó al florecimiento del mercantilismo inglés, a la propagación de las ideas de la libertad de mercado y a la formación del carácter rebelde del antioqueño (Muñoz Cardona y Mayor, 2017, pp. 136-138).

En 1935 las propuestas del gobierno de unificar el sistema universitario colombiano y dar a la Universidad Nacional el derecho exclusivo de otorgar títulos unieron a todos los antioqueños en defensa de la Universidad de Antioquia, considerada por muchos como muy superior a la Nacional. El ministro antioqueño de educación en Bogotá, Luís López de Mesa, ante la propuesta del gobierno nacional afirmó: "Siempre hemos sostenido que, entre los infinitos enemigos mortales de Antioquia en todo el país, ninguno es tan feroz, tan agresivo y tan artero, como el antioqueño que sale de su tierra y logra establecerse con algún brillo en Bogotá" (Melo, 1991, p. 148).

Los crecimientos de la industria nacional, la falta de capacidad del Estado para coordinar la política económica y social y establecer un modelo de desarrollo consensuado (Restrepo, 2011, p. 111), además de los sentimientos de exclusión, dieron pie a las diferencias existentes entre la dirigencia empresarial antioqueña y el gobierno nacional, razones que explican por qué las diferentes asociaciones patronales se gestaron principalmente en Antioquia, tales como: la Sociedad de Agricultores de Colombia (SAC) en 1871, Federación Nacional de Cafeteros (FNC) en 1927, la Asociación Nacional de Industriales (ANDI) en 1944, Federación Nacional de Comerciantes (FENALCO) en 1945, Federación Colombiana de Ganaderos (FEDEGAN) en 1963, Asociación Nacional de Instituciones Financieras (ANIF) en 1974, la Federación de Aseguradores Colombianos (FASECOLDA) en 1976 y el Grupo Empresarial Antioqueño (GEA) en 1978 como afirma Pécaut (1970, p. 60), igualmente citado en (CoenenHuther, 2004, p. 82; Restrepo, 2011). Afirma el ingeniero del grupo industrial Suramericana:

(...) la función que cumplían las diversas asociaciones patronales era velar por los derechos de sus afiliados en un marco de libre empresa; representarlos ante el gobierno, los organismos internacionales y las entidades públicas; rechazar la indebida intervención del Estado e impulsar las iniciativas nacionales que determinen progresos económicos y sociales (Restrepo, 2011, p. 111).

La cuarta hipótesis que busca explicar las diferencias del desarrollo en Antioquia con el país nace de la unión política y empresarial con la iglesia católica. Uno de los principales defensores de esta hipótesis es el sociólogo Jacques CoenenHuther en Sociologie des élites: "una de las mejores formas de apreciar la capacidad de influencia de una élite cualquiera es a través del papel que ella juega en la difusión de una innovación material o sociocultural" (2004, pp. 82, 123). Es decir, al mejoramiento social de la calidad de vida de todos los habitantes. En Antioquia, la lógica que subyace a la unión política y empresarial es el desarrollo local a gran escala, la cual es atravesada por el carácter religioso de los antioqueños. Entre 1940 y 1980 en el Club Unión y en el Club Campestre de Medellín, las élites patronales antioqueñas que frecuentaban estos lugares compartían la misma sensibilidad y reconocían sus necesidades empresariales comunes; lo que les permitía fortalecer relaciones comerciales, y a su vez, pensar en cómo mejorar la de los habitantes del Valle de Aburrá, de tal manera que se pudieran mejorar las relaciones sociales de bienestar -de amor al prójimo-, productivas y de empresa, tal y como lo expone Restrepo (2011, p. 79).

Es desde dicho escenario de observación, afirma Coenen-Huther (2004, pp. 54-55), que se da en Antioquia la asociación de la institucionalidad política y empresarial; antes de finales de la década de los años 80, un gran número de concejales, diputados, gobernadores y alcaldes provenían de las familias de las élites antioqueñas, lo cual facilitó la inversión para el desarrollo territorial del Valle de Aburrá sin el abandono de lo social.

Gracias a la labor desplegada por clérigos y a la conservación de unos valores religiosos, la Iglesia católica tuvo fuerte influencia en la vida social y política de Antioquia y mantuvo relaciones muy próximas con las élites patronales, a través de las cuales se emprendieron proyectos comunes y alianzas en diferentes campos como la educación, la acción sindical y la divulgación de su actividad proselitista. (Restrepo, 2011, p. 96)

La iglesia sirvió a la educación y al ascenso social de muchas personas de bajos recursos, entre ellos Marco Fidel Suárez, quien llegó a ser presidente 
de Colombia por el apoyo de las élites empresariales de Antioquia, so pena de ser hijo natural. Lo que favoreció el desarrollo de la aviación en Antioquia, el desarrollo de las comunicaciones y de las vías terrestres. Argumenta Nicanor Restrepo Santamaría que la iglesia en el auge económico de finales de los años 30 en Antioquia contribuyó a la formación del trabajador responsable, a través del fomento de los buenos hábitos laborales, tales como: disciplina en el manejo del tiempo, permanencia en el trabajo, repetición ininterrumpida de labores. "La mezcla entre paternalismo y religiosidad fue un sistema en el que el patrón asimilo los rasgos de autoridad del padre de familia, revirtiéndolo con un sustento religioso" (Restrepo, 2011, p. 99), en el que el patrón se sentía en el deber moral cristiano de ayudarle y protegerlo. De igual manera, afirma Pécaut:

Dicha posición paternalista no tuvo como fin la explotación avasallante del trabajador, más bien fue inspiradora de una justicia social ejercida a través de diferentes programas de bienestar para los trabajadores en aspectos como la salud, vivienda, educación, aprovisionamiento de los hogares, recreación y atención a la familia (Pécaut, 1970, p. 58).

A principios de 1949, por ejemplo, la ANDI fue promotora de un hospital para los trabajadores y sus familias, el hoy Hospital Pablo Tobón Uribe, terminado en 1954 por Coltabaco y Cervunión. De igual manera, la ANDI y la Arquidiócesis de Medellín en 1954 fueron promotoras del subsidio familiar para los hijos de los trabajadores de bajos ingresos; para la administración de dichos recursos se creó en 1954 la Caja de Compensación Familiar de Antioquia (COMFAMA) en Medellín. Las empresas de textiles emprendieron programas de vivienda para los trabajadores mediante la construcción de barrios cercanos a las instalaciones de las fábricas, tales como Bello, Itagüí, La Milagrosa y Buenos Aires. En cobertura de educación básica, varias empresas abrieron escuelas y centros de formación para esposas e hijos de los trabajadores, idea que copió el gobierno nacional y dio paso a la creación del Servicio Nacional de Aprendizaje (SENA). Incluso algunas empresas abrieron comisariatos exclusivos para los trabajadores y sus familias, allí se ofrecían productos de la canasta familiar a precios subsidiados. El GEA también es impulsor de la cultura, especialmente desde 1978 con el Museo de Antioquia y en 1980 con la creación del Museo de Arte Moderno de Medellín (MAMM).
En el desarrollo de los servicios públicos domiciliarios, la municipalidad de Medellín, a principios del siglo XX fue adquiriendo las diferentes empresas de servicios públicos tanto públicas como mixtas de la región y las agrupó en 1920 en las Empresas Públicas Municipales; en 1955 pasaron a ser Empresas Públicas de Medellín EPM, tal y como lo comentan Restrepo (2011, pp. 102-103) y López en la Revista Administer:

La municipalización de los servicios públicos fue el producto de una acometida de la élite antioqueña representada en el Concejo de Medellín, la cual llegó a la plena conciencia, en la segunda década del siglo XX, de que ciertos servicios esenciales en el devenir urbano no deberían ser ofrecidos por particulares, ni siquiera por compañias mixtas. (2005, p. 75)

En la actualidad EPM representa un poco más del 30\% de los ingresos corrientes del municipio de Medellín y posee un gran reconocimiento nacional e internacional por su excelente administración pública. A través de EPM más del $97 \%$ de los municipios de Antioquia disfrutan de amplia cobertura en servicios de agua y energía, contribuye con la seguridad energética a través de nuevas inversiones en hidroeléctricas y empresas como la interconexión Eléctrica Nacional ISA, a pesar del revés que tuvo el patrimonio antioqueño en el 2016 con la venta de una de sus filiales, ISAGEN, por el gobierno nacional. Venta realizada por el mecanismo de subasta a un único postor, la multinacional canadiense Brookfield Asset Management. En servicios de aseo, las Empresas Varias de Medellín (EMVARIAS) creada en 1964, es una de las mejores del país, contribuyendo con el Área Metropolitana a la sostenibilidad ambiental de la región.

Se concluye que el crecimiento urbanístico de la región ha obedecido a necesidades económicas de expansión de la empresa antioqueña sin estudios propiamente dichos de planeación del territorio, lo que implica la existencia de fenómenos propios de la saturación urbanística de ciudades no pensadas para la gente.

\section{El Área Metropolitana del Valle de Aburrá: ejemplo de unión}

Con el Acto legislativo 01 de 1986 se dio inició en Colombia a una forma más amplia de hacer democracia, la elección popular de alcaldes 
y de gobernadores abrió las puertas a una mayor responsabilidad política de los votantes en el desarrollo local y regional. El voto programático, plasmado en el Artículo 259 de la Constitución Política de Colombia brinda al votante el poder de decidir con responsabilidad ciudadana el futuro de su región. Sin embargo, la asociación política y empresarial para el crecimiento regional no se termina, por el contrario, se redefine al incorporar a las organizaciones sociales y comunales.

El crecimiento económico de la ciudad de Medellín en el siglo XX creó un fenómeno de conurbación en el cual desaparecen las fronteras municipales de los territorios que integran el Valle de Aburrá. Fenómeno de crecimiento que llevó a la conformación del Área Metropolitana en 1980 como institución que coordina la unión municipal para el desarrollo sustentable y la calidad de vida de sus habitantes. El Área Metropolitana financia los proyectos de bienestar común municipal de acuerdo al avalúo catastral alcanzado. Si la región crece, aumentan los avalúos catastrales, que son cobrados en las tasas de contribución e impuestos directos del predial. De esta manera los municipios están autorizados al cobro de la sobretasa ambiental en un $0.15 \%$ según el Artículo 43 de la Ley 99 de 1993, y el 0.2\% de sobretasa metropolitana, Literal a, Artículo 22 de la Ley 128 de 1994.

A partir del informe Monitor Medellín en 1990, la región comenzó a trabajar en sus ventajas comparativas locales, bajo el lema "Ciudades Clúster”, 23 años antes de la promulgación de la Ley 1626 del 2013 de áreas metropolitanas, (Restrepo, 2011; Ospina e.t., 2007, p. 2; Cámara de Comercio de Medellín para Antioquia, 2006, p. 5; Castro, 2013, p. 32).

Dos documentos determinantes fueron los informes Monitor Colombia y Monitor Medellín realizados a principios de la década de 1990, en ellos se esclarecieron las bases sobre las que reposa el desarrollo del país, y se muestran los retos que debe afrontar la región para estar posicionada como un eje productivo y competitivo en el ámbito global. De igual manera, se desarrollaron estudios como: "La Ventaja Competitiva de la Actividad Empresarial Antioqueña hacia el Siglo XXI" y "Antioquia Avanza hacia nuevos Esquemas de Desarrollo Empresarial", entre otros, buscando el desarrollo productivo de la región de manera sostenible y dinámica, frente a Colombia y el mundo. Es en todos estos estudios donde se emp- ieza a vislumbrar y consolidar la iniciativa Clúster como una potente estrategia de desarrollo que permite construir tejido empresarial por medio de la conformación de redes de negocios y la identificación de oportunidades de mercado (Cámara de Comercio de Medellín para Antioquia, 2006, p. 5).

En la conformación y fortalecimiento de las Ciudades Clúster participan las universidades de la región con estudios de investigación en sectores de producción, software, energía, salud, planeación del territorio, sostenibilidad ambiental y convivencia pacífica, tal y como lo afirma Mejía (agosto de 2017). Dichos estudios son financiados e implementados por el sector público y empresarial del departamento. Las Ciudades Clúster se han visto fortalecidas en su capacidad productiva manufacturera con la actividad turística; ejemplo de ello es la Ruta Lechera que con el apoyo de la empresa regional lechera COLANTA, las administraciones municipales y otras empresas turísticas y comerciales de San Pedro de los Milagros, Belmira, Entrerríos, Santa Rosa y Don Matías impulsaron el crecimiento productivo y de empleo de la región. Los municipios de la Ruta Lechera disfrutan de la valorización de las tierras, generadas por la alta actividad turística y comercial, el mejoramiento de las vías de comunicación intermunicipal e infraestructura social en educación, recreación, cultura religiosa y de salud; en otras palabras, en calidad de vida local. Desde el 2014 la Ruta Lechera ha sido integrada al crecimiento urbanístico del Área Metropolitana a través del sistema de transporte masivo integrado Metro.

Otro de los logros de la Ciudad Clúster con la comunidad está en la conformación de los Centros de Desarrollo Empresarial Zonal (CEDEZOS) los cuales alimentan los Parques de Emprendimiento en los diferentes barrios del Área Metropolitana. Los CEDEZOS alimentan las ideas comunitarias de empresa con BANCUADRA y Banco de los Pobres, que prestan a tenderos, madres cabeza de hogar para pago de servicios a partir de la solidaridad de vecinos. De igual manera, los Parques Bibliotecas y las Unidades de Vida Articulada UVA fortalecen el encuentro ciudadano para la educación, la convivencia y la vida en comunidad con equidad. Los CEDEZOS, BANCUADRA, las UVA, Parques Bibliotecas y de Emprendimiento también cuentan con el apoyo financiero de la empresa privada y de las empresas públicas descentralizadas que financian la educación, la cultura y la salud en el Departamento, 
ellas son: EPM, Fábrica de Licores de Antioquia (FLA), Beneficencia de Antioquia y EMVARIAS.

Desde el 2010, la integración regional se estructura con base en la articulación de la planeación intermunicipal para el manejo integrado de los recursos, denominada provincia; ejemplo de lo anterior son Cartama, Sinifaná y Penderisco, San Juan y la de Aguas, Bosques y Turismo. Esta última conformada bajo la Ordenanza 13 del 31 de mayo de 2018. Provincias antioqueñas creadas en conformidad con la Ley 1454 de 2011. La primera Provincia Administrativa y de Planificación (PAP) de Cartama representa una nueva manera de administrar el territorio a partir del reconocimiento de las potencialidades sociales, económicas, culturales y ambientales de las localidades (Departamento Administrativo de Planeación, Gobernación de Antioquia, 2017). Las provincias, al igual que el Valle de Aburrá llevan el nombre del nacimiento de las fuentes naturales hídricas que las atraviesan, como estrategia de identidad cultural. Ello invita a sus habitantes a conservarla y a preservarla (Prieto, 2017). Esta integración fue reglamentada por la Ley de Descentralización Administrativa 1654 de 1995, la Ley Orgánica de Ordenamiento Territorial LOOT 1454 de 2011 y la Ley de Regalías 1530 del 2012.

La asociatividad municipal permite articular la municipalidad en la planificación integral del desarrollo sustentable y sostenible del territorio. Integración que cuenta con la participación activa de las comunidades, a través de las organizaciones sociales y comunales. Dicha asociatividad municipal mejora ostensiblemente el crecimiento económico, la sostenibilidad fiscal, la equidad social, la sustentabilidad ambiental y la competitividad. De esta manera, la conformación de PAP buscan en el mediano plazo garantizar adecuadas condiciones de vida de la población, a través de la articulación de servicios públicos domiciliarios, facilitando la ejecución de proyectos y obras regionales, según lo establece la Ordenanza 54 de 2016 de la Asamblea de Antioquia.

Las PAP son una estrategia de crecimiento conjunto de las subregiones para detener procesos migratorios que acrecientan el fenómeno de conurbación en el Valle de Aburrá y dejan sin capital humano a los municipios por la creciente fuga de jóvenes talentosos que van en busca de mejores oportunidades laborales y de estudio; esto retrasa el crecimiento y empobrece aún más a los municipios; por lo que, el Área Metropolitana y la Gobernación de Antioquia, junto con las Universidades aportan recursos de inversiones para el transporte de mercancías y de conocimientos al fortalecimiento de la descentralización territorial a través de iniciativas de integración administrativa para la planificación local y regional. Por cada peso de inversión que colocan las PAP la Gobernación de Antioquia y el Área Metropolitana ponen otro (Prieto, 2017).

Se concluye que, a partir de 1980, Antioquia comienza a crecer con visión de unión regional y de planeación del territorio, lo que la lleva a la construcción de planes de desarrollo a largo plazo que vincula a todas sus subregiones y compromete a las administraciones públicas locales y a las comunidades en favor del desarrollo futuro de todo el territorio. La primera forma de asociatividad municipal fue el Área Metropolitana del Valle de Aburrá, después le siguieron las provincias de Cártama, San Juan y Sinifaná, Aguas, Bosques y Turismo y la Ruta Lechera.

\section{La importancia del Área Metropolitana en el desarrollo del Valle de Aburrá}

Antes de hablar de lo positivo y de los retos que tiene el Área Metropolitana con la construcción de una ciudad para la gente, comencemos con los reconocimientos internacionales alcanzados por la ciudad. En el marco del concurso internacional City of the Year organizado por Wall Street Journal la ciudad de Medellín y el Área Metropolitana fue reconocida como la ciudad más innovadora en el 2013. En el 2012 The Business Destination Travel Awards de la revista Business Destination a través de estudio de encuestas internacionales, 550 compañias y empresarios eligieron a la gran ciudad del Área Metropolitana como el mejor destino de Suramérica para hacer negocios. De igual manera, el Observatorio Económico del Caribe reconoció en el 2012 a Medellín como la ciudad más competitiva entre las 22 principales ciudades capitales de Colombia.

La unión de las iniciativas de políticas públicas: Ciudades Clúster y Área Metropolitana, logra que, los 10 municipios del Valle de Aburrá adelanten proyectos sustentables que benefician al $80 \%$ de la población, por ejemplo, en torno a la movilidad, tales como: el Metro, creado en 1979 y que inició operaciones el 30 de noviembre de 1995; el Parque Ecoturístico Arví desde el 2007; el Hospital San Vicente Fundación desde el 2011; el Parques 
del Río desde el 2014; Red de Bicicletas Públicas desde el 2014; Cultura Ciudadana o Cultura Metro desde 1988; Mi Río: recuperación y conservación del Río Medellín y su Alumbrado Navideño desde el 2004; Plan básico de Ordenamiento Territorial desde el 2006; más de 500 gimnasios al aire libre de Metro Parque en todo el Valle de Aburrá; Control permanente a la contaminación de las aguas del río del Valle de Aburrá, a las emisiones de gases y niveles de ruido, entre otros, desde 1996.

Por lo anterior la visión del Área Metropolitana es la de ser una región articulada, con oportunidades de desarrollo sustentable para todos sus habitantes, altos niveles de calidad de vida, con una ciudadanía responsable y participativa, que cree y confía en sus instituciones. Para lograrlo urge pensar en nuevas ideas para abordar aspectos cruciales, como diseñar políticas económicas con rostro humano; articular estrechamente las políticas económicas y las sociales; mejorar la equidad $\mathrm{y}$, por último, llevar adelante alianzas virtuosas entre Estado, empresas y sociedad, en todas sus expresiones, para enfrentar la pobreza mejorando el gasto público con transparencia. Todo esto, en un marco que recupere la ética como motor del proyecto de desarrollo y la gobernanza como logro de la sociedad en su conjunto, como lo afirman Mejía (agosto de 2017), Muñoz Cardona (2018, pp. 136-160) y como se referencia en el Plan Integral de Desarrollo Metropolitano (2007, p. 173).

Los recursos alimentarios, energéticos, de recreación, ambientales, entre otros, deben asegurarse de manera equitativa; para ello es necesario equilibrar la interdependencia entre las ciudades, consolidando cambios culturales con relación a la ocupación y uso del suelo e implementando las Directrices Metropolitanas de Ordenamiento Territorial (Plan Integral de Desarrollo Metropolitano, 2007, p. 174; Prieto, 2017).

Para recuperar la ética social y de la administración pública, los líderes sociales deben entender que la inversión social no es asistencialismo, es inversión en infraestructura social: salud, educación, empleo y seguridad hacen parte de la creación de oportunidades de acceso a los beneficios del desarrollo y de generación de espacios que propicien actividades económicas dignas, tal y como lo plantean en sus artículos (Muñoz y Mayor, 2017; Mejía, 2017). Esto espacios son posibles en el marco de las alianzas entre Empresa-UniversidadEstado para la formación académica avanzada, la innovación y el desarrollo tecnológico, y el estímulo a la aplicación de conocimiento en el contexto regional; además, implica un cambio cultural hacia la relación costo-efectividad en la administración pública y asignación de recursos de inversión (Plan Integral de Desarrollo Metropolitano, 2007, p. 174). En otras palabras, garantizar la transparencia en la gestión pública mediante mecanismos claros de control y altos niveles de calidad de la burocracia, y finalmente, controlar que toda acción se desarrolle en armonía con el medio ambiente (Plan Integral de Desarrollo Metropolitano, 2007, p. 175).

En materia de habitabilidad y calidad de vida el Plan de Desarrollo Metropolitano se propone: atención al déficit de espacio público, revirtiendo las condiciones de degradación de la habitabilidad de barrios, que conducen al desarraigo y falta de identificación por parte de los vecinos y usuarios; revertir las causas principales de habitabilidad por contaminación atmosférica; aumento de la superficie ruidosa; pérdida de confort por inadecuado tratamiento de la vegetación y de ventilación en los espacios públicos; aumento de la inseguridad vial y la congestión (Plan Integral de Desarrollo Metropolitano, 2007, pp. 178,179). También es deber prioritario en el Plan de Desarrollo Metropolitano suplir los déficits de calidad de la vivienda, la reubicación de habitantes en zonas de alto riesgo y el aprovechamiento de los bienes de interés cultural y bienes patrimoniales como espacios de interacción social (Plan Integral de Desarrollo Metropolitano, 2007, pp. 179).

El Plan de Desarrollo Metropolitano pretende la construcción de un modelo de ciudad para todos, en el que todos los habitantes de las diez ciudades se sientan incluidos. Esto ha de instrumentarse mediante el concurso efectivo de instituciones organizadas, la integración económica regional, el mercado de trabajo y las organizaciones comunitarias (Plan Integral de Desarrollo Metropolitano, 2007, p. 183). Asimismo, se debe integrar y consolidar la riqueza existente en la biodiversidad de ambientes, desde los más naturales y protegidos hasta los más públicos y urbanos, pasando por el fomento de la noción de ciudad-jardín y la consecuente integración de los espacios privados (Plan Integral de Desarrollo Metropolitano, 2007, p. 185).

Se trata de construir un concepto que va más allá de la redistribución de los ingresos, se habla de justicia en la sociedad; un concepto con- 
temporáneo que involucra la libertad política, la eficiencia económica en el uso de recursos públicos y privados y la razonable satisfacción social (Plan Integral de Desarrollo Metropolitano, 2007, p. 186). Lo anterior se alcanza mejor en un clima de interacción cooperativa en el que el voto, la negociación y las acciones compartidas son precedidas de informes transparentes de gestión y en el que las acciones del gobierno son marcadas por la legitimidad (Plan Integral de Desarrollo Metropolitano, 2007, p. 189). Según Mejía (agosto de 2017) las alcaldías del Área Metropolitana y la gobernación de Antioquia han sido las mejor evaluadas a nivel nacional; incluso han contado con niveles de favorabilidad por encima del $82 \%$ y una evaluación a su gestión de 3,7. La razón es el desarrollo alcanzado en obras de infraestructura de transporte público, mejoramiento de viviendas, calidad en la educación y de cobertura en servicios públicos.

El Área Metropolitana del Valle de Aburrá al igual que las provincias son un modelo administrativo y de planificación; son un esquema asociativo público-privado para el desarrollo regional sostenible, al respecto afirma Eugenio Prieto Soto en la Editorial "Mejor todos juntos" de El Metropolitano del Valle de Aburrá:

El Área avanza en la tarea de hacer realidad la descentralización política, fiscal y administrativa como instrumento para reducir las desigualdades y las inequidades en los territorios y avanzar definitivamente en la búsqueda de un diálogo que rompa las asimetrías entre el centro y las periferias de nuestro territorio (...) la integración es razón de crecimiento local capaz de contrarrestar la soberbia del centralismo administrativo y la desidia de las autonomías locales. $(2017$, p. 2)

La asociación municipal con equidad es un modelo de crecimiento en inversiones para la satisfacción de necesidades comunes, en cuanto a movilidad, infraestructura, servicios públicos, vivienda recreación y esparcimiento. La meta del Plan Integrado de Desarrollo Metropolitano es cumplir con las ejecutorias en más de un 95\% (Ver Tabla 1).

\section{Retos de la asociatividad municipal en Antioquia}

En cuanto al índice de calidad de vida ICV, la pobreza en el 2016 fue del 14,1\%, la más baja desde el año 2007 , lo que fortalece y estimula el trabajo conjunto de líderes sociales y de la ciudadanía con empresarios, academia, y gobierno en pro del bien común de los habitantes del Valle de Aburrá (Ver Tabla 1).

Según el informe de Calidad de Vida, 2300 personas han participado con iniciativas barriales en 168 reuniones para complementar el desarrollo local y otras 1900 personas en 79 encuentros para la construcción de la reglamentación de la planeación del presupuesto participativo (El Colombiano, 15 de julio de 2017, p. 12). El Área pretende mejorar el Îndice de Condiciones de Vida Multidimensional ICVM, optimizando el PIB local; también aspira a disminuir el índice de desigualdad del ingreso y bajar la tasa de desempleo, incrementando el indicador de empresas por cada mil habitantes al pasar de 37 en el 2017 a 50 en el 2019. Para ello, se pretende formalizar 24 mil unidades productivas afirma el economista antioqueño (Muñoz, 2018, p. 522).

Las nuevas fuentes productivas con las cuales la región pretende reducir la desigualdad en ingresos y el desempleo deben enmarcarse bajo la idea de Territorio verde; esta concentra la movilidad inteligente y la construcción sostenible. Los nuevos negocios de empresas deben ser sostenibles, deben contribuir a la eficiencia energética y a la disposición del agua. En otras palabras, deben contribuir a la idea de Región inteligente e innovadora.

La Región inteligente integra la planeación urbana, la gestión inteligente de los servicios públicos y la educación. Se debe fortalecer el clúster de salud en medicina avanzada que integra la tríada universidad-Estado-empresa. Las nuevas fuentes productivas deben ser resultado de Región emprendedora, que integra a la microempresa con el ecosistema e integra al Valle de Aburrá con otras regiones como la provincia de Cártama que integra 11 municipios, Sinifaná y Penderisco 8 municipios, San Juan 6 municipios al suroeste del Departamento de Antioquia y al Oriente del departamento la provincia del Embalse 5 municipios, en conformidad con la Ley Orgánica de Ordenamiento Territorial LOOT 1454 de 2011.

Las Provincias Administrativas de Planificación (PAP) y las Regiones Administrativas de Planificación (RAP) no son entidades para la competencia de los escasos recursos públicos, sino para ser competentes, es decir, para el fortalecimiento institucional y de la región en el desarrollo con equidad humana y territorial (Prieto, 2017, p.2; Departamento Administrativo de Planeación, Gobernación de Antioquia, 2017). Los 
Tabla 1.

Indicadores de condiciones de vida 2008-2020

\begin{tabular}{|c|c|c|c|c|c|}
\hline Metas & $\begin{array}{l}\text { Situación } \\
\text { inicial }\end{array}$ & $\begin{array}{l}\text { Meta } 1 . \\
2010\end{array}$ & $\begin{array}{l}\text { Meta } 2 \\
2012\end{array}$ & $\begin{array}{l}\text { Meta } 3 \\
2016\end{array}$ & $\begin{array}{l}\text { Meta } 4 \\
2020\end{array}$ \\
\hline Límite inferior Yso/N & 2.768USD & 3.200 USD & 3.500 USD & 4.200 USD & 4.700 USD \\
\hline Limite superior Yso/N & & 3.600 USD & 4.000 USD & 5.000 USD & 5.800 USD \\
\hline Límite inferior IDH & 0.78 & 0.78 & 0.8 & 0.83 & 0.84 \\
\hline Límite superior IDH & & 0.8 & 0.83 & 0.85 & 0.86 \\
\hline $\begin{array}{l}\text { Límite inferior Espacio } \\
\text { Público }\end{array}$ & $\begin{array}{l}3.47 \mathrm{M} 2 / \\
\text { hab. }\end{array}$ & $\begin{array}{l}3.8 \mathrm{M} 2 / \\
\text { hab. }\end{array}$ & $\begin{array}{l}4.8 \mathrm{M} 2 / \\
\text { hab. }\end{array}$ & $\begin{array}{l}6.0 \mathrm{M} 2 / \\
\text { hab. }\end{array}$ & $\begin{array}{l}6.6 \mathrm{M} 2 / \\
\text { hab. }\end{array}$ \\
\hline $\begin{array}{l}\text { Límite superior Espacio } \\
\text { Público }\end{array}$ & & $\begin{array}{l}3.9 \mathrm{M} 2 / \\
\text { hab. }\end{array}$ & $\begin{array}{l}4.9 \mathrm{M} 2 / \\
\text { hab. }\end{array}$ & $\begin{array}{l}6.1 \mathrm{M} 2 / \\
\text { hab. }\end{array}$ & $\begin{array}{l}6.7 \mathrm{M} 2 / \\
\text { hab. }\end{array}$ \\
\hline $\begin{array}{l}\text { Límite inferior Espacio } \\
\text { Público Verde }\end{array}$ & $\begin{array}{l}5.68 \mathrm{M} 2 / \\
\text { hab. }\end{array}$ & $\begin{array}{l}6.2 \mathrm{M} 2 / \\
\text { hab. }\end{array}$ & $\begin{array}{l}7.5 \mathrm{M} 2 / \\
\text { hab. }\end{array}$ & $\begin{array}{l}8.4 \mathrm{M} 2 / \\
\text { hab. }\end{array}$ & $\begin{array}{l}9.4 \mathrm{M} 2 / \\
\text { hab. }\end{array}$ \\
\hline $\begin{array}{l}\text { Límite superior Espacio } \\
\text { Público Verde }\end{array}$ & & $\begin{array}{l}6.6 \mathrm{M} 2 / \\
\text { hab. }\end{array}$ & $\begin{array}{l}7.9 \mathrm{M} 2 / \\
\text { hab. }\end{array}$ & $\begin{array}{l}8.8 \mathrm{M} 2 / \\
\text { hab. }\end{array}$ & $\begin{array}{l}9.9 \mathrm{M} 2 / \\
\text { hab. }\end{array}$ \\
\hline $\begin{array}{l}\text { Límite inferior Áreas } \\
\text { Críticas Contaminadas }\end{array}$ & 8,74 & & & & \\
\hline $\begin{array}{l}\text { Límite superior Áreas } \\
\text { Críticas Contaminadas }\end{array}$ & & & & & 0.0 \\
\hline $\begin{array}{l}\text { Límite inferior } \\
\text { Satisfacción social con } \\
\text { los Gobiernos Locales }\end{array}$ & & $70 \%$ & $72 \%$ & $75 \%$ & $80 \%$ \\
\hline $\begin{array}{l}\text { Límite superior } \\
\text { Satisfacción social con } \\
\text { los Gobiernos Locales }\end{array}$ & & $75 \%$ & $77 \%$ & $80 \%$ & $85 \%$ \\
\hline
\end{tabular}

Fuente: (Departamento Administrativo de Planeación, Gobernación de Antioquia, 2017; Plan Integral de Desarrollo Metropolitano, 2007; Plan de Gestión, 2015). El ingreso per cápita anual (Yso/N) está en dólares norteamericanos. El Índice de Desarrollo Humano IDH se mide en una escala de 0 a 1 según el Programa de las Naciones Unidas para el Desarrollo PNUD. Porcentaje de áreas críticas por contaminación ambiental. Superficie susceptible de recuperación ambiental por mal estado de los recursos como el aire 2.877,33 hectáreas y con suelos erosionados 87,62 hectáreas; lo que equivale al 8,74. Evaluación de satisfacción de la sociedad con los gobiernos locales, calidad de la gestión pública en el Valle de Aburrá. La respuesta a la pregunta de satisfacción es: Excelente, Muy Bueno, Bueno, Regular, Malo. Entre 0\% y 59\% se considera que el desempeño es Bajo. Entre 60\% y 79\% se considera que el desempeño es Normal. Entre el $80 \%$ y el 100\% se considera que el desempeño es Alto (Plan Integral de Desarrollo Metropolitano, 2007).

territorios no se ordenan sólo a través de decisiones políticas sino de la vinculación de todas las fuerzas sociales que la integran; de allí que la nueva ruta del desarrollo está enmarcada por la asociatividad de los territorios como herramienta para la planeación con criterios de sostenibilidad con equidad (Prieto, 2017, p.2; Muñoz, 2018). Una planeación que consulta las realidades, culturales, económicas, productivas y de competitividad. Actualmente el Área ha logrado consolidar 48 acuerdos y decretos municipales relacionados con desarrollo económico local sostenible (Prieto, 2017).

El Plan Estratégico Metropolitano de Ordenamiento Territorial (PEMOT), reúne los Planes 
de Ordenamiento Territorial POT de cada uno de los 10 municipios del Área Metropolitana. El PEMOT intenta mejorar la urbanización y la movilización de los habitantes; se estima que el 58,5\% del total de la población antioqueña vive en el Área Metropolitana; de allí la necesidad de todos los municipios de trabajar unidos en bien del ordenamiento territorial a través del PEMOT. Esta institución fue creada en el 2013 con funciones comunes como calidad del agua, vías, transporte masivo, espacio público, clasificación de suelos y densidades habitacionales. En las misionales se consideran las siguientes líneas estratégicas: planeación y gestión para la equidad; calidad ambiental y desarrollo sostenible; movilidad a través del transporte público sostenible, segura y amable y seguridad, convivencia y paz, tal y como lo establece el (Plan de Gestión, 2015, p. 36).

El documento final del PEMOT que prepara al Valle de Aburrá para su desarrollo futuro e integración con los Valles del Oriente y del Occidente, fue presentado a la junta del Área Metropolitana en marzo del 2018 para su aprobación e implementación. Entre los retos y oportunidades que plantea el Área Metropolitana como respuesta asociativa frente a la coordinación del desarrollo humano y territorial, articulada a programas locales, regionales, nacionales y de integración mundial se encuentra este documento final del PEMOT en el Plan de Gestión (2015); en cuatro líneas misionales, dos transversales, diecinueve componentes y treinta seis programas (Plan de Gestión, 2015, pp. 6, 12). Todo este sistema de planificación con un único objetivo: desarrollo con justicia en la sociedad.

El desarrollo sostenible implica atender los problemas de conurbación, principalmente los ambientales en: expansión territorial no controlada de las ciudades; deterioro de los ecosistemas estratégicos regionales; excesos de consumo de agua, energía, suelo y recursos naturales renovables; inseguridad en el abastecimiento de alimentos y materias primas provenientes del sector primario; contaminación del aire, agua y suelo por emisiones, vertimientos y residuos sólidos $\mathrm{y}$ líquidos; escasez, baja accesibilidad y deterioro del espacio público y paisaje urbano; localización de viviendas y población en general, en zonas de alto riesgo; desintegración y gestión fragmentada de los modos y sistemas de transporte, tanto nacionales como regionales, metropolitanos y municipales (Plan de Gestión, 2015, p. 286) ${ }^{2}$.

De acuerdo a la Tabla 2, por cada operación estratégica que compete al Área Metropolitana, desde lo misional y lo normativo, se plantea los siguientes subproblemas específicos que deberán ser atendidos y corregidos para alcanzar las metas planteadas al 202033 (Plan de Gestión, 2015, p.p. 193-194).

Planeación. Planear para reducir la inequidad humana y territorial, las deficiencias de hábitat afectivo $\mathrm{y}$ efectivo del territorio; las deficiencias en la articulación de programas y coordinación del desarrollo armónico, integrado, sostenible y sustentable del Área Metropolitana. En lo empresarial planear para mejorar la logística de transporte terrestre, marítimo, fluvial y aéreo de carga (Ver Tabla 2).

Calidad ambiental. Planear con las universidades para reducir el deterioro de la calidad ambiental; la falta de articulación, concertación y sostenibilidad en el uso, aprovechamiento de recursos naturales por parte de la sociedad e insuficiencia en el cumplimiento de las competencias del Área Metropolitana del Valle de Aburrá como autoridad ambiental (Ver Tabla 2).

Movilidad. Mejorar la articulación municipal, los diálogos, las concertaciones y la cultura ciudadana, que generan pertinencia con el territorio. Es menester, incluso culturizar a los inmigrantes de otras regiones del país que adolecen de cultura ambiental y de respeto ciudadano (Ver Tabla 2).

Seguridad, la convivencia y la paz. Mejorar la capacidad de respuesta del Área Metropolitana e instituciones de control ante recurrentes informalidades, ilegalidades y violencias en la ciudad-región; pero, ante todo, una política municipal de conciliación y prevención a delitos menores entre vecinos (Ver Tabla 2).

Soporte institucional, gestión y cooperación. Los diferentes municipios metropolitanos deben hacer acciones conjuntas tendientes a solucionar problemáticas asociadas a la inequidad social y territorial y una deficiencia en la gestión de recursos para cerrar las brechas de inequidad social territorial en educación y salud. Deben exigir a otros departamentos y a la nación cumplimientos

2 Ver Tabla 2.

3 Ver Tabla 1. 
Tabla 2.

Componentes y Programas: Líneas misionales y transversales.

\begin{tabular}{|c|c|c|}
\hline Linea estratégica & Componente & Programa \\
\hline \multirow{2}{*}{$\begin{array}{l}\text { Planeación y } \\
\text { gestión para la } \\
\text { equidad }\end{array}$} & Región Metropolitana & $\begin{array}{l}\text { 1. Planeación metropolitana integral para la articulación } \\
\text { regional. } \\
\text { 2. Planeación corporativa para el fortalecimiento institucional. } \\
\text { 3. Intervenciones integrales del modelo de ordenamiento } \\
\text { territorial }\end{array}$ \\
\hline & $\begin{array}{l}\text { Integración social y } \\
\text { territorial }\end{array}$ & $\begin{array}{l}\text { 4. Gestión social, educación y cultural } \\
\text { 5. Equipamiento y espacios públicos sostenibles. } \\
\text { 6. Estrategia territorial metropolitana de vivienda y hábitat. } \\
\text { 7. Desarrollo económico, innovación y turismo sostenibles. } \\
\text { 8. Promoción y coordinación de la prestación racional de los } \\
\text { 9. Estrateos públicos a nivel metropolitano. } \\
\text { 9. Estrateritorial de salud pública. }\end{array}$ \\
\hline \multirow{3}{*}{$\begin{array}{l}\text { Calidad ambiental } \\
\text { y desarrollo } \\
\text { sostenible }\end{array}$} & Control y vigilancia & $\begin{array}{l}\text { 10. Fortalecimiento de la autoridad ambiental urbana y } \\
\text { colaboración interinstitucional para la gestión territorial } \\
\text { rural. }\end{array}$ \\
\hline & Gestión ambiental & $\begin{array}{l}\text { 11. Biodiversidad y servicios Eco sistémicos. } \\
\text { 12. Producción y consumo sostenibles. } \\
\text { 13. Gestión integral de residuos sólidos } \\
\text { 14. Gestión integral del recurso hídrico } \\
\text { 15. Calidad del aire, prevención y control de la contaminación } \\
\text { atmosférica }\end{array}$ \\
\hline & Gestión del riesgo & $\begin{array}{l}\text { 16. Conocimiento y reducción del riesgo } \\
\text { 17. Manejo del desastre, gobernanza, educación y } \\
\text { comunicación del riesgo. }\end{array}$ \\
\hline \multirow{5}{*}{$\begin{array}{l}\text { Movilidad } \\
\text { sostenible, segura y } \\
\text { amable. }\end{array}$} & $\begin{array}{l}\text { Movilidad no } \\
\text { motorizada }\end{array}$ & $\begin{array}{l}\text { 18. Infraestructura segura e incluyente. } \\
\text { 19. Sistema de bicicletas públicas } \\
\text { 20. Promoción, educación y cultura de la movilidad }\end{array}$ \\
\hline & $\begin{array}{l}\text { Integración del } \\
\text { transporte público }\end{array}$ & $\begin{array}{l}\text { 21. Mejoramiento de la calidad y cobertura de transporte } \\
\text { público } \\
\text { 22. Integración del transporte público } \\
\text { 23. Fortalecimiento y modernización empresarial del transporte } \\
\text { público }\end{array}$ \\
\hline & $\begin{array}{l}\text { Logistica y transporte } \\
\text { de carga }\end{array}$ & 24. Organización logística del territorio \\
\hline & Seguridad vial & 25. Plan metropolitano de seguridad vial con visión "0" (cero). \\
\hline & Conectividad física & 26. Mejoramiento de la conectividad de la infraestructura vial. \\
\hline \multirow{9}{*}{$\begin{array}{l}\text { Seguridad, } \\
\text { convivencia y paz }\end{array}$} & Seguridad & 27. Gestión de la seguridad metropolitana \\
\hline & Convivencia & 28. Promoción de la convivencia para la integración social \\
\hline & Paz y posconflicto & 29. Construcción de paz territorial \\
\hline & Gestión institucional & $\begin{array}{l}\text { 30. Fortalecimiento de la institucionalidad metropolitana } \\
\text { 31. Mejoramiento y modernización institucional. }\end{array}$ \\
\hline & $\begin{array}{l}\text { Gestión } \\
\text { metropolitana para } \\
\text { territorios inteligentes }\end{array}$ & 32. Fortalecimiento de la capacidad de gestión metropolitana. \\
\hline & $\begin{array}{l}\text { Cooperación y } \\
\text { alianzas }\end{array}$ & 33. Generación de alianzas sinergias para la cooperación. \\
\hline & $\begin{array}{l}\text { Coherencia } \\
\text { organizacional }\end{array}$ & $\begin{array}{l}\text { 34. Direccionamiento estratégico de la comunicación para el } \\
\text { fortalecimiento y posicionamiento institucional. }\end{array}$ \\
\hline & Información pública & $\begin{array}{l}\text { 35. Creación y sostenimiento de una agencia metropolitana de } \\
\text { información pública }\end{array}$ \\
\hline & Movilización social & $\begin{array}{l}\text { 36. Dinamización de la participación ciudadana en el ámbito } \\
\text { metropolitano. }\end{array}$ \\
\hline
\end{tabular}

Fuente: Plan de Gestión, 2015, p.p.197-198. 
en las garantías de coberturas mínimas de su población; ya que muchos de los jóvenes niños y personas adultas que son atendidos en salud y educación en el Área Metropolitana del Valle de Aburrá provienen de territorios muy pobres y con altos niveles de corrupción pública (Ver Tabla 2).

Comunicación pública y movilización social. Mejorar los procesos de comunicación pública (informativos, deliberativos y movilizadores) para convocar y articular la voluntad y acción comprometida de los diferentes actores involucrados en el desarrollo del Valle de Aburrá (Ver Tabla 2).

\section{Conclusiones}

Si bien antes de 1986 el crecimiento de la región era determinado por las necesidades del mercado y de las empresas que buscaban ser competitivas, sin una planeación del territorio y un compromiso de sus gentes o sociedad civil; después de 1986 el desarrollo de la región ha obedecido a una planeación estratégica del territorio a largo plazo, la cual ha contado a su vez con la unión empresarial público-privada -partnership-, en la que la sociedad civil es el jugador sombra más importante para la construcción del territorio. La sociedad civil es el actor político, que a través de las organizaciones sociales y comunales locales inciden en el crecimiento y desarrollo económico de la región con sostenibilidad ambiental.

Antioquia crece porque sus sectores empresariales y políticos trabajan unidos en pos del bienestar común de todos los ciudadanos con equidad y justicia en lo social, como afirma el gerente de Área Metropolitana (Prieto, 2017). Muestra de ello son las provincias de Cártama, Sinifaná, San Juan, de Aguas, Bosques y Turismo; las cuales se han conformado con tres fines específicos: primero, crear cultura ambiental para la preservación de los recursos naturales e hídricos, de allí que sus nombres obedezcan al nombre de los ríos que las atraviesan o circundan; segundo, ir rompiendo las fronteras invisibles de la politiquería para integrarse administrativamente con fines de crecimiento económico y bienestar social para todos. Para tal efecto el Área Metropolitana aporta el $66 \%$ de cada proyecto de inversión de los municipios asociados en Provincia. Tercero, el Área Metropolitana busca detener las inmigraciones de antioqueños de municipios cercanos que profundizan en los fenómenos típicos de las conurbaciones por la falta de oportunidades económicas, de salud y de estudio.

Con todo lo anterior, los 10 municipios del Área Metropolitana son innovadores en cultura política y de innovación social. Formas de organización e integración administrativa que han beneficiado a 50 municipios en mejores oportunidades de empleo, estudio y de salud dentro de sus mismos territorios. No significa lo anterior, que en Antioquia y en Área Metropolitana del Valle de Aburrá, no exista desigualdad o inequidad, significa que la región trabaja para cambiar la cultura del clientelismo político propiciando bienestar empresarial y social; de allí, el legendario arraigo del antioqueño a su territorio (Muñoz, 2018). Significa, de acuerdo a informes del rector de la Universidad EAFIT, que los antioqueños creen más en sus gobernadores, alcaldes y concejos. Significa que las inversiones se ven reflejadas en el crecimiento y desarrollo de la ciudad y en los niveles de cobertura, por lo que evalúan la corrupción regional en menor porcentaje al nacional (Mejía, 2017).

\section{Referencias}

Bejarano, J.A. (1984). "La Economía”. Manual de Historia de Colombia. Bogotá: Instituto Colombiano de Cultura, Tomo III, pp. 17-77.

Brew, R. (2000). El desarrollo de Antioquia desde la independencia hasta 1920. Medellín: Universidad de Antioquia.

Botero, M.M. (1985). Instituciones Bancarias en Antioquia. 1872-1886. Medellín. Revista Lecturas de Economía, No 17., pp. 45-147.

Cámara de Comercio de Medellín para Antioquia. (2006). Clúster una estrategia para crear ventaja competitiva (Documentos Comunidad Clúster No. 1). Medellín: Taller de Edición.

Cámara Comercio de Medellín para Antioquia. (2017). Estadísticas de la composición empresarial. Medellín: Cámara de Comercio de Medellín para Antioquia.

Cámara de Comercio del Aburrá Sur. (2017). Estadísticas de la composición empresarial. Sabaneta: Cámara de Comercio Aburrá Sur.

Castro, F. (2013). 20 Años de políticas de competitividad en Colombia. Bogotá: Fedesarrollo.

CEPAL y GTZ. (2000). La gestión del desarrollo en Antioquia, Colombia. Santiago de Chile: CEPAL 
Coenen-Huther, J. (2004). Sociologie des élites. Paris: Armand Colin.

DANE. (2015). Proyecciones demográficas poblacionales. Bogotá: Departamento Nacional de Estadística.

Departamento Administrativo de Planeación, Gobernación de Antioquia. (2017). Informe final de las propuestas de implementación acordadas en los talleres de aprendizaje sobre las provincias en Italia y su aplicación en la provincia administrativa y de planificación -PAP- de Cártama. Medellín.

El Colombiano. (15 de julio de 2017). Metro: Informe Ciudad. Informativo noticioso.

Londoño, C.F. (2004). Grupo empresarial antioqueño. Evolución de políticas y estrategias 1978, 2002. Revista Escuela de Ingeniería de Antioquia, No 1 , pp. 47-65.

López, J.C. (2005). La gestión de la empresa pública: lecciones de una empresa de servicios públicos. Revista Administer, (7), pp. 70-80. Mejía, J. (agosto de 2017) Un camino hacia la integridad. Encuentro Nacional de Concejales. Encuentro llevado a cabo en Medellín, Colombia.

Melo, J. (1991). "La política antioqueña 1904-1946". Historia de Antioquia. Medellín: Suramericana de Seguros. Segunda reimpresión. Artículo pp. 143-175.

Muñoz Cardona, À.E., y Mayor, P. (2015). The SME or MPYME in Latin America, Japan, European Union, United States and the Cluster in Colombia. International Journal of Humanities Social Sciences and Education (IJHSSE), 2 (6), pp. 1-16.

Muñoz Cardona, Á.E. (2009). Macroeconomía y desarrollo. Medellín: Ude@, programa de formación continuada, Universidad de Antioquia, Facultad de Ingeniería.
Muñoz Cardona, Á.E., y Mayor, P. (2017). Las Juntas de Acción Comunal del Municipio de Itagüí en el desarrollo local. Revista Administración \& Desarrollo, (1), pp. 49-66.

Muñoz Cardona, Á. E. (2018). Descubriendo la economía. ¿Cómo lograr crecimiento y bienestar en economías locales? Bogotá, Escuela Superior de Administración Pública, ESAP, Facultad de Investigaciones.

Ospina, T., Ospina, O., y Velásquez, J.A. (2007). Comisión Tripartita: Acuerdo de Voluntades. Medellín: Cámara de Comercio de Medellín para Antioquia, Alcaldía de Medellín y Área Metropolitana del Valle de Aburrá.

Pécaut, D. (1970). Entrepreneurs, syndicalisme et pouvoir politique local: le cas de Medellin. Paris: Recherche Coopérative, Villes et Régions en Amérique Latine, No 1, p.p. 57-68.

Plan Integral de Desarrollo Metropolitano. (2007). Hacia la integración regional sostenible 2008-2020. Un Plan del Área Metropolitana del Valle de Aburrá. Medellín: Oficina Asesora del Área de Comunicaciones Área Metropolitana del Valle de Aburrá, METROPOLI.

Plan de Gestión. (2015). Territorios integrados 2016-2019. Área Metropolitana Valle de Aburrá. Medellín: Oficina de Planeación del Área Metropolitana del Valle de Aburrá.

Prieto, E. (2017). La fuerza de la asociatividad. El Metropolitano del Valle de Aburrá. Editorial, Área Metropolitana Valle de Aburrá.

Restrepo, N. (2011). Empresariado antioqueño y sociedad, 1940-2004: influencia de las élites patronales de Antioquia en las políticas socioeconómicas colombianas. Medellin: Universidad de Antioquia, Colección Clio. 